\title{
Demonstration of wave optic in physics education
}

\section{Hao Lv, Aimei Liu, Shengyi Zhang, Yongjun Xiao}

Hao Lv, Aimei Liu II, Shengyi Zhang III, Yongjun Xiao IV, "Demonstration of wave optic in physics education," Proc. SPIE 10452, 14th Conference on Education and Training in Optics and Photonics: ETOP 2017, 104524H (16 August 2017); doi: 10.1117/12.2267929

SDIE Event: 14th Conference on Education and Training in Optics and Photonics, ETOP 2017, 2017, Hangzhou, China 


\title{
Demonstration of wave optic in physics education
}

\author{
Hao Lv*, Aimei Liu, Shengyi Zhang and Yongjun Xiao \\ The Key Laboratory of electric light source and lighting, School of Physics and Electronic \\ information engineering, Hubei Engineering University, Xiaogan 432000, P.R.China
}

\begin{abstract}
The set has been designed for simple and clear demonstration of wave optics basic principles. Laser diode as highly collimated light source is used as a basic element of the laser ray box. That way the quality of teaching improves and offers us new possibilities in comparison with the classic incandescent lamp. Moreover the demonstration of beams passing through the optical elements is possible. Such a solution enables the effective demonstration and modeling of basic optical devices. A classic light source cannot be used for such a range of examples. Another remarkable advantage arises from very low demand for room light conditions. Wave optic demonstration set using laser ray box can be used in standard classrooms without any additional room darkening.
\end{abstract}

Keywords: Wave optic, physics education, demonstration

\section{INTRODUCTION}

Optic is a main content in physics education and the wave optics is an important point of optic to explain some phenomena, such as interference and diffraction of light, it is necessary to go beyond geometrical optics ${ }^{1-3}$. But many students understand and master the wave optics difficultly; it is mainly because that the wave optics has the certain abstractness $s^{4-5}$. What 'more, the optical experiments should be operated in the daytime, otherwise, the phenomena of wave optics are difficult to operate and observe in the optical experiment because of the complex operations and the interference of background light. Accordingly, how to help the students understand the wave optics becomes very important. In this paper, a new demonstration instrument of the wave-optic which can be used to demonstrate some phenomena such as interference and diffraction of light is given. Compared with conventional optical instruments, it is easy to operate and don't need the daytime. Accordingly, it is beneficial to understanding knowledge of the wave-optic for the students in the everyday teaching..

\section{DESIGN}

Figure 1 gives the structural diagram of the demonstration instrument of the wave optics. It is mainly composed of a battery 、 Laser diodes $(650 \mathrm{~nm}$ and $532 \mathrm{~nm})$ and the screens which have a multi-slit、a crossing-grating 、 a single-slit、a pinhole and a one-dimensional grating in Fig.1(a). The corresponding photo of the demonstration instrument of the wave optics is shown in Fig.2 (b).

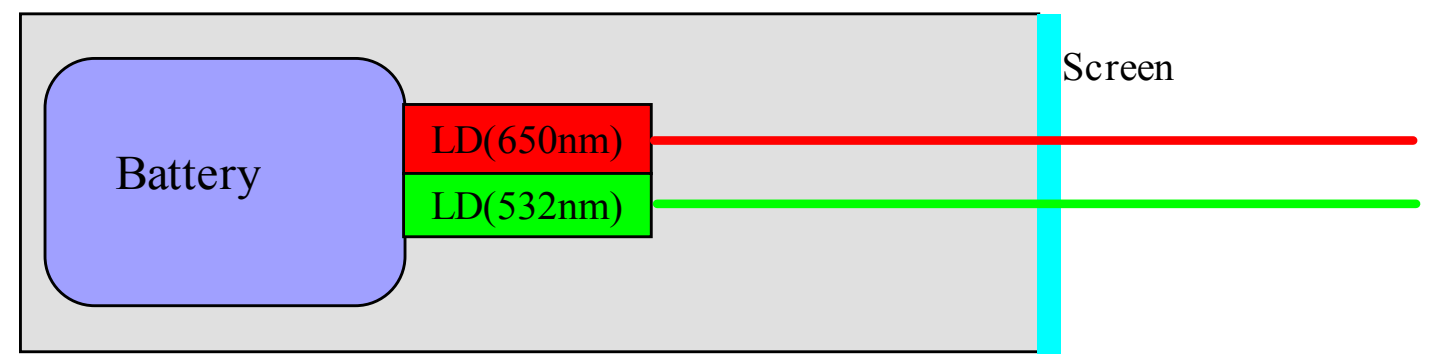

Figure 1. Structural diagram of the demonstration instrument of the wave optics

*kuerlvhao@hotmail.com

14th Conference on Education and Training in Optics and Photonics: ETOP 2017, edited by Xu Liu,

Xi-Cheng Zhang, Proc. of SPIE Vol. 10452, 104524H · C 2017 ICO, IEEE, OSA, SPIE

CCC code: $0277-786 X / 17 / \$ 18 \cdot$ doi: $10.1117 / 12.2267929$

Proc. of SPIE Vol. $10452104524 \mathrm{H}-1$ 

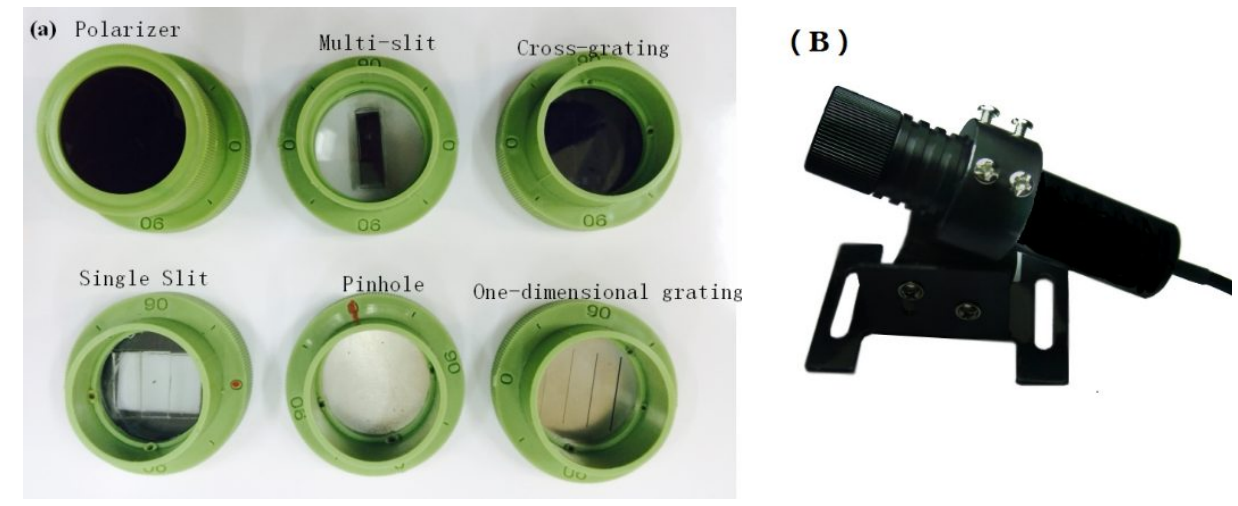

Figure 2.Demonstration instrument of the wave optics

\section{RESULTS AND DISCUSSION}

\subsection{Single-slit diffraction}

Fig. 3 gives the diffraction images of the different width of the single slits which sizes are $0.05 \mathrm{~mm} 、 0.1 \mathrm{~mm} 、 0.2 \mathrm{~mm}$ and $0.4 \mathrm{~mm}$ respectively. It can be seen from the diffraction images that the width of the diffraction fringe is is proportional to the laser wavelengths when the width of the single slit is a constant. Taking into account the condition for the central bright fringes ${ }^{6}$

$$
\Delta x_{0}=2 \frac{D}{a} \lambda
$$

Where $D 、 a$ and $\lambda$ are the distance, the width of the single slit and the lase wavelength respectively. When the values of $D$ and $a$ are $5 \mathrm{~m}$ and $0.1 \mathrm{~mm}$, the widths of the central bright fringes are $6.5 \mathrm{~cm}$ and $5.32 \mathrm{~cm}$ respectively under the different laser wavelengths
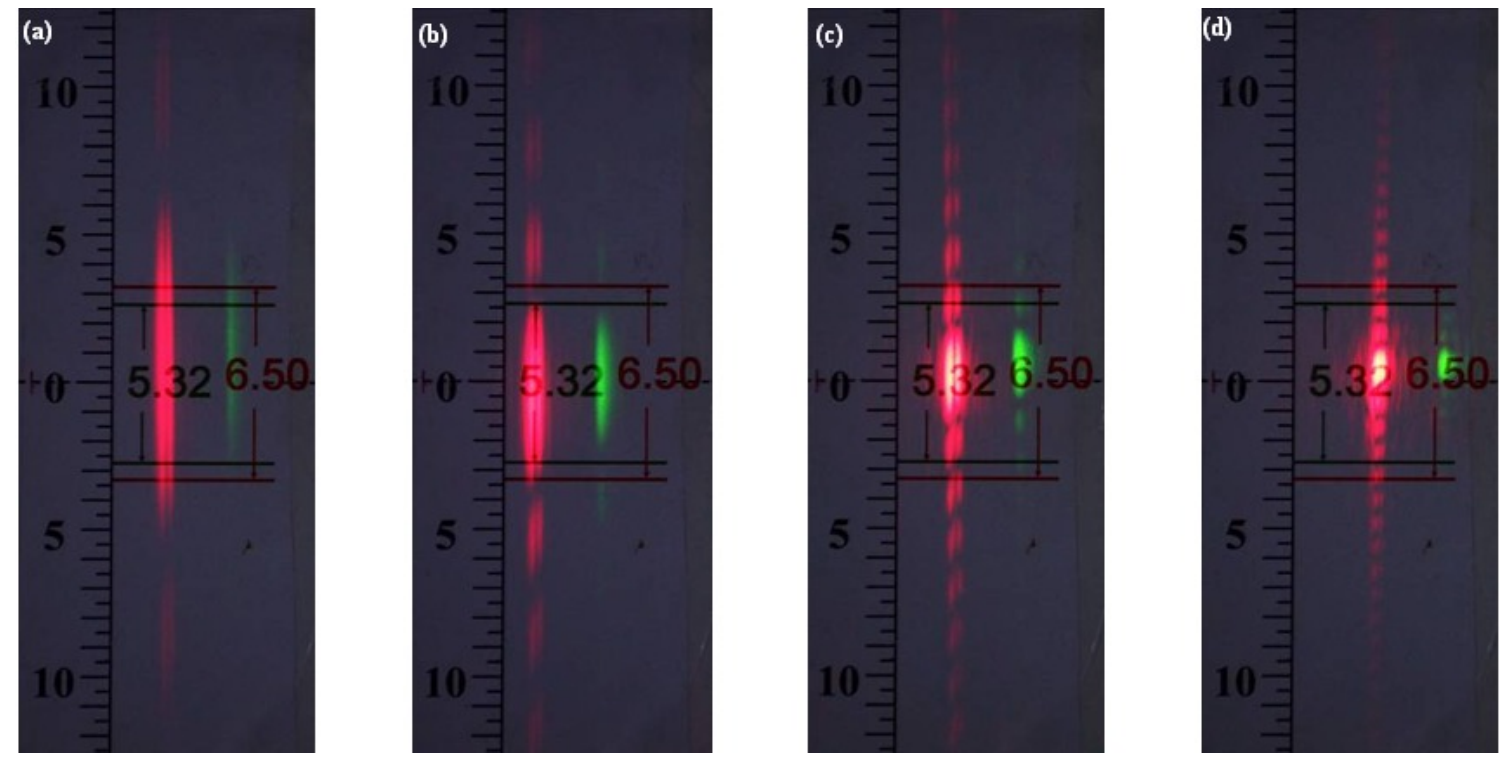

Figure 3. Diffraction images of the different width of the single slits (a: $0.05 \mathrm{~mm} ; \mathrm{b}: 0.1 \mathrm{~mm} ; \mathrm{c}: 0.2 \mathrm{~mm} ; \mathrm{d}: 0.4 \mathrm{~mm}$ ) 


\subsection{Pinhole diffraction}

The diffraction images of the pinholes which the pinholes diameters are $0.4 \mathrm{~mm}$ and $0.2 \mathrm{~mm}$ respectively are shown in Fig.4. With increasing the pinholes diameters the diffraction images of the pinholes becoming more and more obvious. Fig. 5 gives the diffraction images of the triangle pinholes which side length is $0.2 \mathrm{~mm}$ and the rectangular aperture which side length is $0.4 \mathrm{~mm} \times 0.2 \mathrm{~mm}$. Compared with the triangle pinholes, it can be seen that the diffraction phenomenon can be more easily observed from the rectangular aperture.
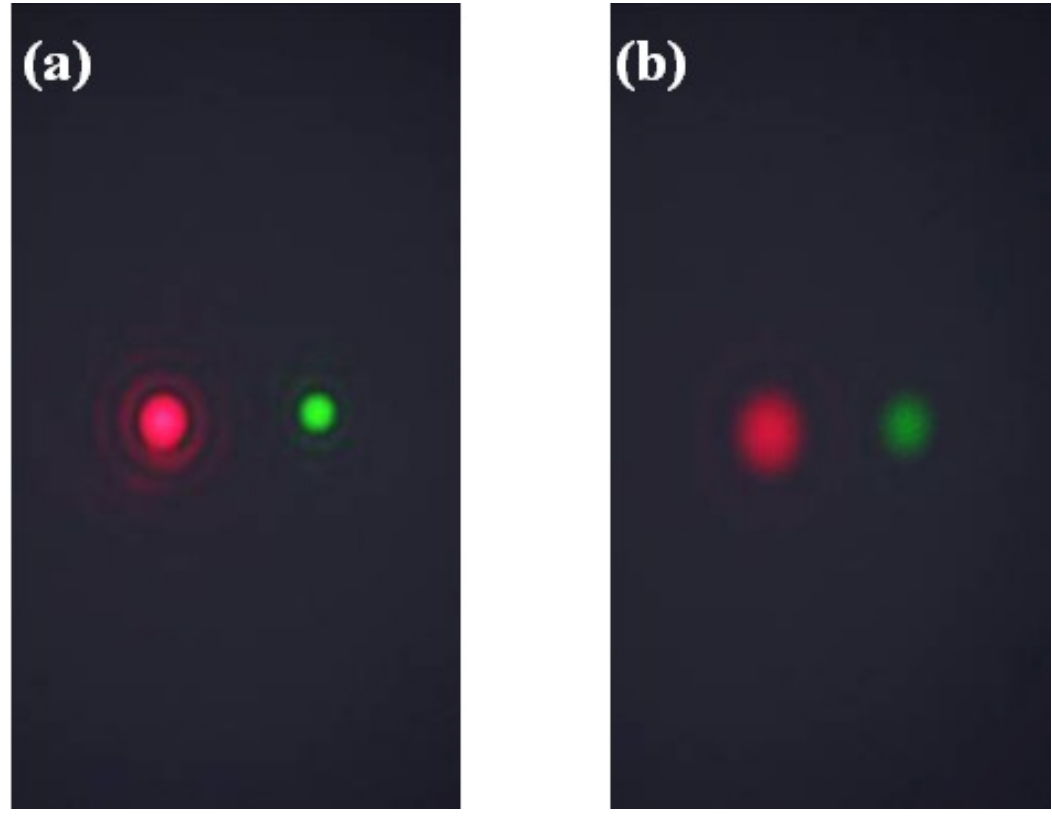

Figure 4. Diffraction images of the pinholes having the different diameters (a: $0.4 \mathrm{~mm} ; \mathrm{b}: 0.2 \mathrm{~mm}$ )
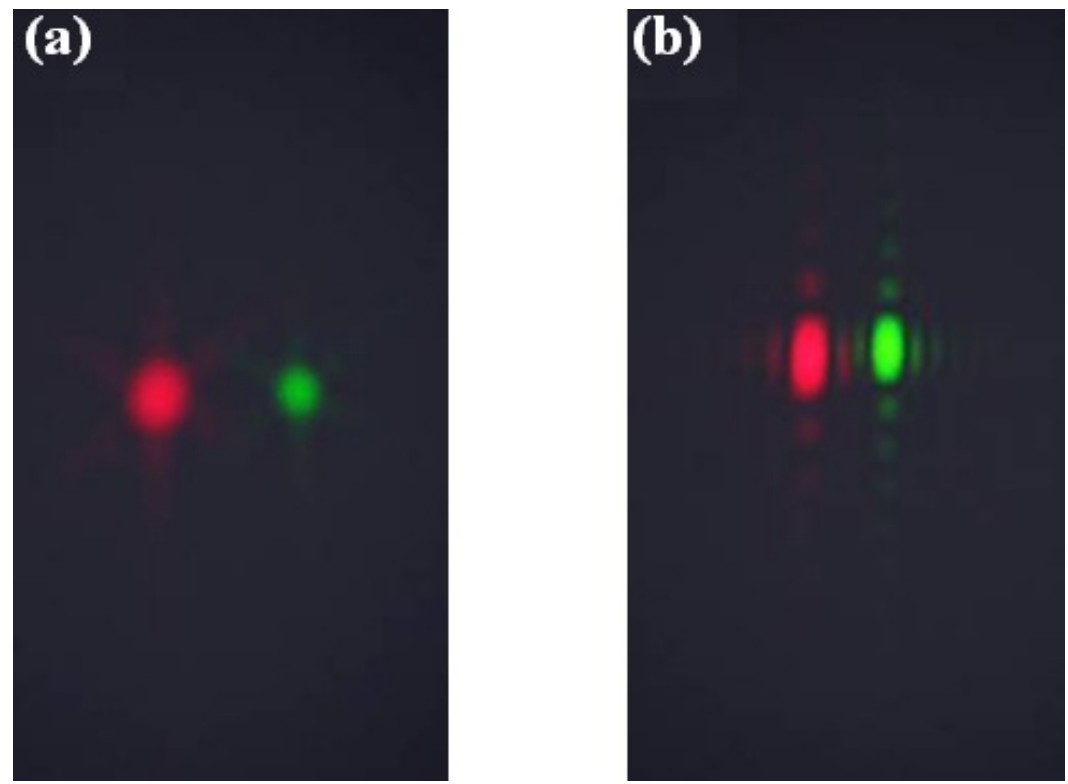

Figure 5. Diffraction images of the triangle pinholes which side length is $0.2 \mathrm{~mm}(\mathrm{a})$ and the rectangular aperture which side length is $0.4 \mathrm{~mm} \times 0.2 \mathrm{~mm}(\mathrm{~b})$ 


\subsection{One dimensional-grating and the crossing-grating diffraction}

Figs. 6 and 7 give the diffraction images of the one-dimensional grating and the crossing-grating. The red green and two-beam laser diffractions from left to right on the same grating are shown in Fig.6(a). In Fig.6 (b), it shows the diffraction phenomena when the different gratings which the line densities are $100 \mathrm{~L} / \mathrm{mm} 、 300 \mathrm{~L} / \mathrm{mm}$ and $600 \mathrm{~L} / \mathrm{mm}$ from left to right are illustrated with the two laser beams, and indicates the diffraction stripes increase gradually with the line densities increasing. In Fig.7, the line densities of the crossing-gratings are $0.06 \mathrm{~mm} 、 0.08 \mathrm{~m}$ and $0.1 \mathrm{~mm}$ from left to right individually. It can be seen that the line densities has little effect on two-beam laser diffractions.
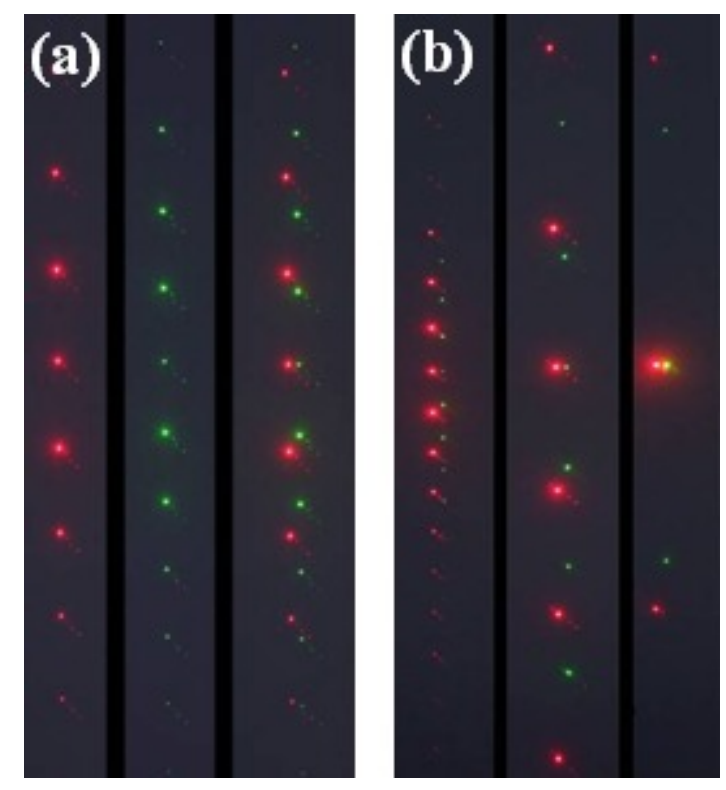

Figure 6. Diffraction images of the one-dimensional grating
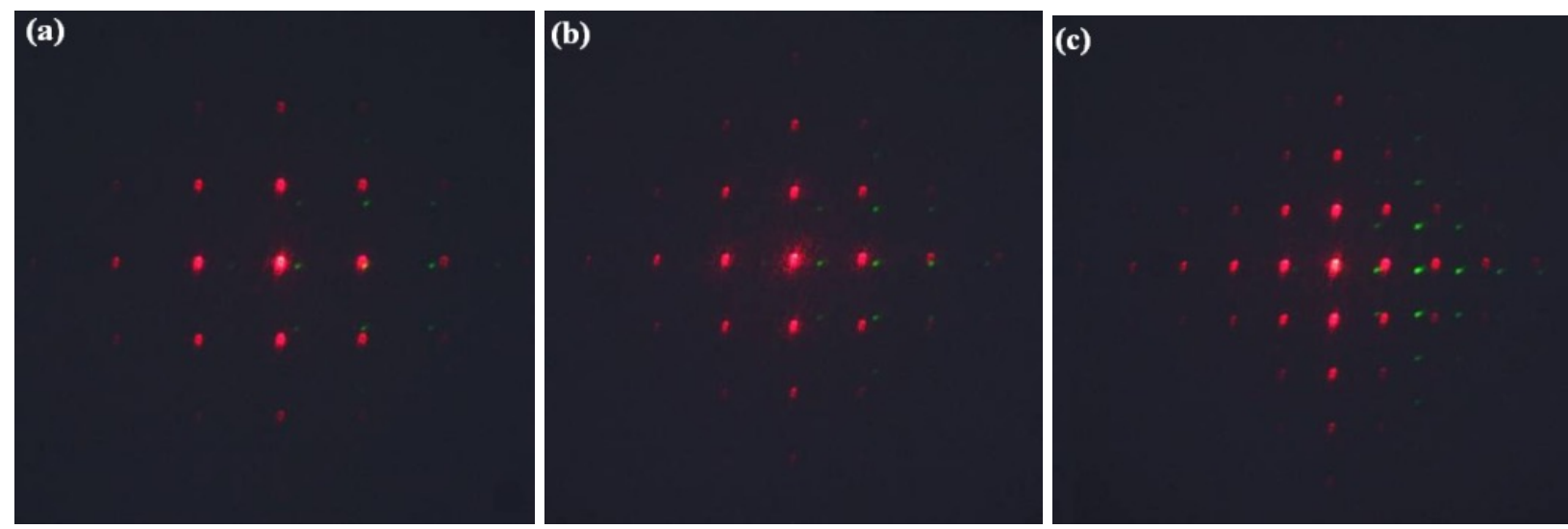

Figure 7. Diffraction images of the crossing-grating

\section{CONCLUSIONS}

In this paper, a demonstration device is produced in order to be used observe diffraction phenomena, such as Single-slit v pinhole、One dimensional-grating and the crossing-grating diffractions. The set can serve the demo for general classroom and demonstrate the diffraction phenomena on wave-optics. It helps students to comprehend the concept and to get more perceptual knowledge of wave-optics in the physics education. 


\section{ACKNOWLEDGMENTS}

This research was supported by the Research Foundation of Education Bureau of Hubei Province (Grant No. 2014378, 2016398), and Hubei Engineering University (Grant No.2014A26). We would like to thank Professor Guangwu Yang for providing the instrument of the wave optics.

\section{REFERENCES}

[1] Ersoy, O. K., [Diffraction, Fourier Optics and Imaging], John Wiley \& Sons Press, New Jersey,177-187(2007)

[2] Menn, N., [Practical Optics],Elsevier Academic Press, San Francisco, 64(2004)

[3] Born, M., Wolf, E., Bhatia, A. B., Clemmow, P. C., Gabor, D., Stokes, A. R., Taylor, A. M., Wayman P. A. and Wilcock, W. L., [Principles of Optics: Electromagnetic Theory of Propagation, Interference and Diffraction of Light],Cambridge University Press, 45-65 (1999)

[4] Stavroudis, O. N.,[The Mathematics of Geometrical and Physical Optics],John Wiley \& Sons Press, New Jersey, 67-78(2007)

[5] Minsung, K., Byoung, J.K., Hwan Hong, L., Krishnamoorthy P.and Myoungsik, C.,[Demonstration of a Standing Light Wave with a Laser Pointer], Journal of the Korean Phy.Soc.56, 1542( 2010).

[6] Römer, H. ,[Theoretical Optics], John Wiley \& Sons Press, New Jersey, 167(2007) 